\title{
EXPONENTIAL DECONTAMINATION MODELS FOR COUNT DATA
}

\author{
RICHARD G. CORNELL* and ASHOK K. BANSAL** \\ Florida State University, Tallahassee, Florida, U.S.A.
}

(Received 28 December, 1970)

\begin{abstract}
Several models are developed for the estimation of the rate of exponential die-off from decontamination data. Calculations with illustrative data are reported which indicate that the estimation of this rate and its variance are sensitive to changes in modelling assumptions. Since extrapolation using this estimated rate is used in the specification of planetary quarantine standards, special care should be taken in the selection of an appropriate model and corresponding estimation procedure for the analysis of each set of decontamination data to be used for this purpose.
\end{abstract}

\section{Introduction and Summary}

This report is concerned with the development of decontamination models based upon the assumption that the probability, $\theta_{t}$, that a single micro-organism is alive at time $t$ after initial exposure to a decontamination procedure is exponential, that is, is of the form

$$
\theta_{t}=\mu^{t}, \quad 0<\mu<1 .
$$

Observations are assumed to consist of counts $x$ of the number of organisms alive per unit of test material and corresponding sampling or dilution fractions, $d$. It is common practice to report only the ratios $y=x / d$, which are the estimated total counts that would have resulted if complete counts had been feasible.

The $y$ ratios, or the $x$ and $d$ values if they are recorded separately, are used to estimate $\mu$. The negative inverse of the logarithm to the base ten of this estimate is called the $D$-value by microbiologists. Its importance, and hence the importance of accurate and precise estimation of $\mu$, in the specification of space quarantine standards is emphasized in Section 2. The remainder of the paper is concerned with the development of models based on (1.1) which enable the estimation of $\mu$ under a variety of assumptions. For each model $\mu$ is estimated using the data of Section 3 in order to illustrate the importance of choosing a model and a corresponding method of analysis which incorporate the most realistic assumptions.

The first model presented in Section 4 is the one assumed, at least implicitly, when unweighted least squares calculations are applied to the paired values of $t$ and $v \equiv \log y$. An assumption of the model is that the $v$ values have equal variance, or equivalently when the $x$ 's are similar enough to warrant the assumption that they have equal means, that the $x$ values also have equal variance. The estimation of the variance of the estimate of $\mu$ is sensitive to departures from this assumption in the illustration.

* Now at the University of Michigan, Ann Arbor, Michigan, U.S.A.

** Now at Delhi University, Delhi, India. 
An alternative model is presented in Section 5 which utilizes both the $x$ and $d$ values instead of their ratios and which is based on independent binomial distributions for the $x$ counts. For our illustrative data this model leads to a maximum likelihood estimate of $\mu$ similar to the least square estimate for the first model but the estimated variance of this second estimate is considerably less. This shows the importance of dealing with the observed $x$ counts instead of the derived counts, if possible, and of avoiding the assumptions of constant variance of the $v$ logarithms when it is unrealistic as it is shown to be for our example.

A Poisson approximation to the binomial model is presented in Section 6. Similar estimates of $\mu$ and equal variances of these estimates are calculated for these two models. The advantages of this Poisson model are that it leads to simpler maximum likelihood estimation calculations when $n$ is not assumed known than does the binomial model and that it is more easily extended to the situation where $n$, the initial loading, varies for different exposure times. This extension is devloped in Section 7. For our illustration the estimate of $\mu$ is quite different when $n$ is assumed to fluctuate widely than when it is relatively stable, again illustrating the importance of making realistic initial assumptions.

For all but the binomial model an estimate of $\mu$ is obtained both with $n$, or its mean when $n$ is variable as in Section 7, assumed known and when it is estimated from all the data. In the former case the common practice is followed of setting $n$ equal to $y_{0}$, the $y$ value based on an initial count with $t=0$, and the estimate of $\mu$ is obtained using the remainder of the observations. In the latter situation all of the observations, including $y_{0}$, are used in the same manner to estimate both $n$ and $\mu$. The two estimates of $n$ and the corresponding estimates of $\mu$ differ considerably for each model. This illustrates that $n$ should not be experimentally determined and then assumed known unless it is well determined, that is, unless repeated initial counts have been made.

The assumption of exponential die-off as given by (1.1) is basic to all of the models developed herein and is an assumption often made in analyzing decontamination data. The most common departure is for the rate of die-off, that is, the parameter $\mu$, to change with exposure time $t$. One way to overcome this difficulty is to apply Equation (1.1), and the models derived from it, over only part of the range of $t$. For instance, rapid early die-off is often ignored and models such as those presented here are then used to describe the data when the rate of die-off is more stable.

Another approach for dealing with departures from assumption (1.1) is to assume a different distribution of the underlying tolerances to exposure of the microorganisms being studied. Assumption (1.1) can be thought of as an assumption that the probability a single microorganism dies before time $t$ is $1-\mu^{t}$. This is the cumulative tolerance distribution of a single-parameter exponential distribution.

Thus assumption (1.1) is equivalent to an assumption of exponentially distributed tolerances in the population of microorganisms. The well-known probit and logit methods of analysis have been developed and extensively applied for the more flexible two-parameter normal and logistic tolerance distributions, respectively. Another possible tolerance distribution which is a direct extension of $(1.1)$ is the two-parameter 
gamma distribution. Research is certainly needed to determine appropriate tolerance distributions for a variety of organisms and types of exposure. However, assumption (1.1) is widely used and holds in many experimental situations, so it is appropriate to concentrate in this report on models derived from that assumption.

\section{Importance to Space Quarantine}

Decontamination models are of general interest in microbiology. They are of particular interest in the development of procedures to achieve a high probability of sterilization, for instance, in the food and medical supply industries. However, the main concern which motivated this work is the sterilization of interplanetary spacecraft. International planetary quarantine agreements have been reached which imply that the probability of contaminating Mars must be less than $10^{-r}$ for a single flight, where $r$ is of the order of magnitude of three. The number of viable contaminants on a spacecraft before final decontamination can be made less than $10^{s}$, where $s$ is no larger than eight. So it is pertinent to set $n_{0}$, the number of viable organisms assumed to be present at the beginning of decontamination, equal to $10^{s}$ and to determine the exposure time, say $t_{r}$, needed to reduce the probability that the corresponding $x$ is zero to $1-10^{-r}$. For the models in this paper, it is appropriate to set $t_{r}=(r+s) \delta$, where

$$
\delta=-1 /\left(\log _{10} \mu\right)>0
$$

can be defined from (1.1) as the value of $t$ for which $\theta_{t}=0.1$. An estimate of $\delta$, usually called a $D$-value, can be used to form an estimate $\hat{t}_{r}$ of $t_{r}$ given by

$$
\hat{t}_{r}=(r+s) D \text {. }
$$

The simplicity of (2.2) explains the practice of microbiologists of thinking in terms of the estimator $D$ of $\delta$ instead of in terms of an estimator of $\mu$. Moreover, the use of (2.2) can be thought of as plotting the expected value of $n$ evaluated at $\delta=D$ verses $t$ on semi-logarithmic graph paper and then extrapolating linearly to find the value of $t$ for which this line reaches the sterilization probability standard. The extent of the extrapolation required, which is specified by the multiplier $(r+s)$ in $(2.2)$ and which we have indicated could be as large as eleven, means that it is imperative for sterilization applications to estimate $\delta$, or alternatively $\mu$, as well as possible. Once an estimate of $\mu$, denoted by $\hat{\mu}$, is calculated, the corresponding $D$-value can be computed by replacing $\mu$ by $\hat{\mu}$ in (2.1).

\section{Illustrative Data}

The parameter $\mu$ will be estimated using each of the models presented in this paper for the data presented in Table I. The $t$ and $y$ values listed there have been taken from a graph presented by Bruch (1965) in a discussion of dry-heat sterilization at a Spacecraft Sterilization Technology Conference. It has been assumed that the $y$ values were determined by diluting the test material for each $t$ value so that a count of the order of magnitude of 300 or less could be obtained, making such a count and expanding that count by division by the dilution fraction. Counts and dilution fractions which 


\section{TABLE I}

Number ( $\mathrm{x}$ ) of Bacillus subtilis var. Niger spores recovered per strip vs exposure time $(t)$ in hours at $120^{\circ} \mathrm{C}$ temperature in air at atmospheric pressure

\begin{tabular}{llllrl}
\hline $\begin{array}{l}\text { Trial } \\
\text { number }\end{array}$ & $t$ & $y$ & $d$ & $x$ & $m$ \\
\hline 0 & 0 & $6.3 \times 10^{5}$ & $10^{-4}$ & 63 & $6.3 \times 10^{1}$ \\
1 & 0.167 & $4.6 \times 10^{5}$ & $10^{-4}$ & 46 & $6.3 \times 10^{1}$ \\
2 & 0.500 & $3.4 \times 10^{5}$ & $10^{-4}$ & 34 & $6.3 \times 10^{1}$ \\
3 & 0.750 & $2.5 \times 10^{5}$ & $10^{-4}$ & 25 & $6.3 \times 10^{1}$ \\
4 & 1.0 & $1.6 \times 10^{5}$ & $10^{-3}$ & 161 & $6.3 \times 10^{2}$ \\
5 & 1.5 & $3.4 \times 10^{4}$ & $10^{-2}$ & 336 & $6.3 \times 10^{3}$ \\
6 & 2.0 & $3.4 \times 10^{3}$ & $10^{-2}$ & 34 & $6.3 \times 10^{3}$ \\
7 & 3.0 & $1.4 \times 10^{2}$ & 1 & 136 & $6.3 \times 10^{5}$ \\
8 & 4.0 & $3.4 \times 10^{1}$ & 1 & 34 & $6.3 \times 10^{5}$ \\
9 & 5.0 & $2.0 \times 10^{\circ}$ & 1 & 2 & $6.3 \times 10^{5}$ \\
\hline
\end{tabular}

could have led to the values graphed by Bruch have been entered in the $x$ and $d$ columns, respectively, for illustrative purposes. The $m$ column lists values of $m=d n$ for $n$ set equal to $6.3 \times 10^{5}$, the initial expanded count recorded by Bruch before exposure of his test organisms to dry heat. The first column of trial numbers in Table I indexes the exposure times used in the experiment.

\section{A Least Squares Model}

A simple model can be obtained by noting from (1.1) that $\log \theta_{t}=t \log \mu$. Since $y / n$ is an estimate of $\theta_{t}$, where $n$ is the number of organisms exposed initially, $v=\log y$ satisfies, at least approximately,

$$
v=\log n+t \log \mu .
$$

The initial loading $n$ is assumed to be the same for each $t$. Moreover, it is assumed that the variance of $v$ is the same for each $t$. This latter assumption allows the simple unweighted least squares technique to be applied to $(v, t)$ pairs to estimate the slope $\log \mu$ and, if it is not assumed know, the intercept $\log n$. The former estimate is the negative inverse of the $D$-value when logarithms to the base ten are used.

The assumption of equal variability for the $v$ 's is not entirely unreasonable. Each $x$ is of the same order of magnitude, say $\gamma$. Assume in addition that each $x$ is a sample from the same population with mean $\gamma$. Also assume that each $x$ value has variance $\gamma$ as it would for the Poisson distribution. With these assumptions, the mean and variance of $y$ are $\gamma / d$ and $\gamma / d^{2}$ and the variance of $v=\log y$ is approximately proportional to $1 / \gamma$, a constant independent of $d$ and $t$. The proportionality constant is unity if natural logarithms were used in defining $v$.

The least squares estimate of $\log \mu$ is

$$
\overparen{\log \mu}=\sum u v / \sum u^{2}
$$


with $u=(t-\bar{t})$ if $n$ is unknown, where $\bar{t}$ denotes the sample mean of the $t$ 's. If $n$ is assumed known, $u=t$ and $v$ is replaced by $w \equiv \log y-\log n$. In the former case, the least squares estimate of $\log n$ is

$$
\overparen{\log n}=\bar{v}-(\overparen{\log \mu}) \bar{t}
$$

where $\bar{v}$ is the sample mean of the $v$ 's. In both cases the variance of the least squares estimate of $\log \mu$ is

$$
V(\overparen{\log \mu})=V(v) / \sum u^{2}
$$

where $V(v)$ is the variance of $v$ and is estimated by summing the squared deviations of the $v$ 's from their estimated values calculated by substituting least squares estimates into the right side of the Equation (4.1). This sum of squares is then divided by the number of entries in the sum less the number of least squares estimates calculated.

After use of (4.4), the estimated variance of $\hat{\mu}=\operatorname{antilog}(\overparen{\log \mu})$ is computed by noting that

$$
V(\hat{\mu}) \approx \hat{\mu}^{2} V(\overparen{\log \mu})\left(\log _{e} 10\right)^{2}
$$

when common logarithms are used. The factor $\left(\log _{e} 10\right)^{2}$ on the right of (4.5) is deleted when dealing with natural logarithms.

Using all the data in Table I in Equations (4.2) and (4.3) leads to the estimates $\hat{\mu}=0.069$ and $\hat{n}=1.1 \times 10^{6}$. Setting $n=y_{0}=6.3 \times 10^{5}$ and using Equation (4.2) excluding $y_{0}$ yields $\hat{\mu}=0.082$. The estimated variances of $\hat{\mu}$ for these two situations, calculated from (4.4) and (4.5), are $0.66 \times 10^{-4}$ and $0.61 \times 10^{-4}$, respectively. These illustrative calculations show that somewhat different estimates of $\mu$ can be obtained when $n$ is estimated from all the data. This difference is particularly noteworthy in view of the extrapolation based on $\mu$ to be made in the space quarantine setting as described in Section 2.

In the calculation of the estimated variances of $\hat{\mu}$, estimated variances of $v$ utilizing natural logarithms and deviations of the $v_{i}$ from their estimates were calculated and equalled 0.35 and 0.53 according to whether or not $n$ was set equal to $y_{0}$. If instead the variance of $v$ were estimated, in accord with our justification for assuming that the $v$ 's have common variance, by $1 / \bar{x}$, where $\bar{x}$ is the sample mean of the $x$ 's and an estimate of $\gamma$, then we would obtain 0.011 in both cases as our estimate of the variance of $v$. The disparity between these estimates indicates that the assumption of constant variance for $v=\log y$ is unreasonable for our illustrative data and therefore that none of these variance estimates are very meaningful. However, one of the first pair of variances would be used, if any, since they are based on observed deviations of the $v$ 's from their estimates and not just on modelling assumptions and because the $x$ values are not usually available when least squares calculations are applied to the $y$ 's. This comparison of variance estimates illustrates the fact that the assumption of equal variance for the $v$ 's upon which commonly used unweighted least squares calculations are based can not be taken for granted. 


\section{A Binomial Model}

The reasonableness of the model given by (4.1) depends on the assumption that each $x$ observation has the same mean. This assumption is often unacceptable even though the $x$ 's are usually within a power of ten of each other. A more reasonable assumption, and one which leads to unequal variances for the $v$ 's and to a slightly more complicated model and estimation equations, is that $x$ follows a binomial distribution with each of $d n$ organisms observed at time $t$ independently having probability $\mu^{t}$ of survival. This leads to a model specified by the binomial probability function $f(x)$ for $x$, where

$$
f(x)=\left(\begin{array}{c}
d n \\
x
\end{array}\right)\left(\mu^{t}\right)^{x}\left(1-\mu^{t}\right)^{d n-x}, \quad x=0,1, \ldots, d n,
$$

and where $\left(\begin{array}{c}d n \\ x\end{array}\right)$ is the binomial coefficient representing the number of ways of selecting $x$ organisms from a total available of $d n$.

The model given by Equation (5.1) with both $n$ and each of the sampling fractions assumed known is often encountered in bioassay and epidemiology as documented by Cornell and Speckman (1967) who reference, illustrate and compare several methods for estimating $\mu$. Peto (1953) works out the maximum likelihood procedure which when applied to the data in Table I with $n=y_{0}$ yields $\hat{\mu}=0.078$, which is very close to the comparable least squares estimate of 0.082 given in Section 4 . This method entails maximizing the joint likelihood function which equals the products of the right-sides of Equation (5.1) formed using all of the combinations of $d, t$ and $x$ values observed in the experiment. The approximate (asymptotic) variance formula for $\hat{\mu}$, which is also given by Peto, leads to an estimated variance of $\hat{\mu}$ of $0.17 \times 10^{-5}$. This is considerably smaller than the corresponding variance estimate cited in Section 4 and illustrates, at least when $n$ is assumed known, that taking into account that the $x$ variables do not all have the same mean and using the actual $x$ observations and corresponding dilution factors $d$ instead of just their $y$ ratios makes more efficient estimation of $\mu$ possible. The main drawback to the use of this binomial model instead of the least squares model of Section 4 is the difficult computations required to estimate both $n$ and $\mu$ using all the data. This difficulty is overcome to some extent in the next section.

\section{A Poisson Model}

Since $\theta_{t}$ is usually small relative to $n d$ for $t>0$ because of the exponential die-off assumed in Equation (1.1), the binomial probability model given by (5.1) can alternatively be approximated using the Poison probability function

$$
f(x)=e^{-\lambda} \lambda^{x} / x !, \quad x=0,1, \ldots,
$$

where $\lambda=n d \theta_{t}=n d \mu^{t}$ is the mean of $x$ given $t$ for the distribution specified by (6.1) as well as by (5.1). This model leads to easier estimation calculations than the binomial model in Section 5, particularly when $n$ as well as $\mu$ is to be estimated. 
The model given by (6.1) has been introduced by Williams (1961) in the context of a genetic study for equally spaced $t$ values. He develops tables to assist in the maximum likelihood estimation of $\mu$ and studies the significance of departures from the model. To apply his work directly to the model depicted by (6.1), the dilution factors $d$ would have to be the same for each $t$.

A formula which can be solved iteratively for a maximum likelihood estimate of $\mu$ in model (6.1) for any set of exposure times, thus avoiding the restrictions in William's model, is

$$
n \sum d_{i} t_{i} \hat{\mu}^{t_{i}}-T=0
$$

where $T=\sum t_{i} x_{i}$ and $n$ is assumed to be known. When $n$ is not known and $X \equiv \sum x_{i}$, joint maximum likelihood estimates of $\mu$ and $n$ are given by

$$
\begin{aligned}
& \left(\sum t_{i} d_{i} \hat{\mu}^{t_{i}} / \sum d_{i} \hat{\mu}^{t_{i}}\right)-T / X=0 ; \\
& \hat{n}=X / \sum d_{i} \hat{\mu}^{t_{i}} .
\end{aligned}
$$

A method of solving such equations iteratively which also leads to variance estimates is discussed, for instance, by Rao (1952, pp. 165-172).

For the data in Table I with $n=y_{0}$, Equation (6.2) yields $\hat{\mu}=0.077$. Its estimated variance is $\hat{\mu}^{2} / \sum m_{i} t_{i}^{2} \hat{\mu}^{t_{i}}=0.17 \times 10^{-5}$. These results are almost the same as those obtained with the binomial model in Section 5. When $n$ is not assumed to equal $y_{0}$, Equations (6.3) and (6.4) lead to $\hat{\mu}=0.056$ and $\hat{\mu}=1.4 \times 10^{6}$. These results indicate the sensitivity of the estimate $\hat{\mu}$ to the value taken for $n$ and also the discrepancies which can arise between an estimate of $n$ based on a single initial count and an estimate based upon all the data. This emphasizes again the need for several observations at $t=0$ if $n$ is to be experimentally determined and then assumed known in further calculations used to estimate $\mu$.

\section{A Model with Variable Loadings}

The Poisson model in Section 6 forms a base for easily allowing for the variation in the initial loading $n$ over the tubes prepared for different exposure times. Since $n$ is large in spacecraft decontamination applications, it is reasonable to approximate its distribution by that of a continuous ransom variable and since $n$ can not be negative, the gamma distribution is the natural one to use. Its probability density function, with the mean and variance of $n$ equal to $\alpha / \beta$ and $\alpha / \beta^{2}$, respectively, is

$$
f(n)=\beta^{\alpha} n^{\alpha-1} e^{-\beta n} / \Gamma(\alpha), \alpha, \beta, n>0 .
$$

The gamma distribution is flexible, assuming an exponential form when $\alpha=1$ and moving through stages of decreasing skewness towards normality as $\alpha$ increases.

Multipling the right sides of Equations (6.1) and (7.1) leads to

$$
f(x, n)=\beta^{\alpha}\left(d \mu^{t}\right)^{x} e^{-n\left(\beta+d \mu^{t}\right)} n^{x+\alpha-1} /[\Gamma(\alpha) x !] .
$$

Integrating out $n$ yields a negative binomial probability function for $x$ given by

$$
f(x)=\frac{\Gamma(x+\alpha)}{\Gamma(\alpha) x !}\left(\frac{\beta}{\beta+d \mu^{t}}\right)^{x}\left(\frac{d \mu^{t}}{\beta+d \mu^{t}}\right)^{x}, \quad x=0,1, \ldots, \alpha, \beta>0 .
$$


The mean of $x$ is equal to $\alpha d \mu^{t} / \beta$, which is just the mean $\lambda$ for the binomial and Poisson models of Sections 5 and 6 with $n$ replaced by its mean $\alpha / \beta$.

The parameter $\mu$ has been estimated using (7.2) with the mean $\alpha / \beta$ of $n$ set equal to $y_{0}$, the assumed known and fixed value of $n$ used in calculations for previous models. The parameter $\beta$ has been set successively equal to $1, \frac{1}{2}$, and $0.4 \times 10^{-5}$. When $\beta=1$ the variance and mean of $n$ are equal, as when $n$ has a Poisson distribution. Taking $\beta=\frac{1}{2}$ makes the variance of $n$ twice its mean, representing slightly more variability than when $n$ has a Poisson distribution. When $\beta=0.4 \times 10^{-5}$ the variance of $n$ is much larger than its mean and this illustrates the use of this model when there is extremely diffuse information about $n$. For these three situations the maximum likelihood estimates of $\mu$ are 0.078 for $\beta=1$ and $\frac{1}{2}$, as compared to 0.077 for the corresponding Poisson model in Section 6 , and 0.089 for $\beta=0.4 \times 10^{-5}$. This illustrates that allowing for variability in $n$ does not affect the estimate of $\mu$ when this variability is relatively small but does when it is large, and hence should be taken into account in this latter instance through the use of model (7.2) as opposed to those given by (5.1) and (6.1).

The parameters $\alpha$ and $\beta$ as well as $\mu$ in (7.2) can be regarded as unknown and estimated from the data by the maximum likelihood procedure, although the calculations are formidable. For our illustration, the resultant estimates of $\mu, \alpha$ and $\beta$ are 0.064 , 4.10 and $0.273 \times 10^{-5}$, respectively. The latter two estimates lead to $1.5 \times 10^{6}$ and $5.5 \times 10^{26}$ as the estimated mean and variance of $n$. The point values of these estimates should not be emphasized too much because the likelihood contour is relatively flat around its maximum point, that is, the estimates of $\mu, \alpha$ and $\beta$ could be varied quit a bit without reducing the likelihood of the sample markedly. However, these estimates do indicate that for this example it is not very reasonable to assume that $n$ is the same for each exposure time in the Poisson model in Section 6 or in the binomial model in Section 5 which it approximates. This calculation, like the first one in this section, also illustrates the sensitivity of the estimate of $\mu$ to assumptions made about $n$ in developing the model upon which the estimation calculations are based.

\section{Acknowledgements}

Support for this work was received from grant number NGR-10-004-029 from the National Aeronautics and Space Administration. Partial support for the Florida State University Computing Center, whose facilities were used extensively, was received from the National Science Foundation.

\section{References}

Bruch, C. W.: 1965, Spacecraft Sterilization Technology, National Aeronautics and Space Administration Special Publication SP-108, Washington, D.C., 207-229.

Cornell, R. G. and Speckman, J. A.: 1967, Biometrics 23, 717-737.

Peto, S.: 1953, Biometrics 9, 320-335.

Rao, C. R.: 1952, Advanced Statistical Methods in Biometric Research, John Wiley and Sons, Inc., New York.

Williams, E. J.: 1961, Biometrics 17, 584-606. 